\title{
Infiltrating macrophages in diabetic nephropathy promote podocytes apoptosis via TNF-a-ROS-p38MAPK pathway
}

\author{
Yinfeng Guo ${ }^{1}$, Zhixia Song ${ }^{1}$, Min Zhou ${ }^{1}$, Ying Yang ${ }^{1}$, Yu Zhao ${ }^{1}$, Bicheng Liu ${ }^{1}$ and \\ Xiaoliang Zhang ${ }^{1}$ \\ ${ }^{1}$ Institute of Nephrology, Zhong Da Hospital, Southeast University School of Medicine, Nanjing, Jiangsu 210009, China \\ Correspondence to: Xiaoliang Zhang, email: tonyxlz@163.com \\ Keywords: macrophage, podocyte, apoptosis, diabetic nephropathy \\ Received: July 04, $2016 \quad$ Accepted: May 22, $2017 \quad$ Published: June 07, 2017 \\ Copyright: Guo et al. This is an open-access article distributed under the terms of the Creative Commons Attribution License 3.0 \\ (CC BY 3.0), which permits unrestricted use, distribution, and reproduction in any medium, provided the original author and source \\ are credited.
}

\section{ABSTRACT}

Macrophage infiltration has been linked to the pathogenesis of diabetic nephropathy (DN). However, how infiltrating macrophages affect the progression of DN is unknown. Although infiltrating macrophages produce pro-inflammatory mediators and induce apoptosis in a variety of target cells, there are no studies in podocytes. Therefore, we tested the contribution of macrophages to podocytes apoptosis in DN. in vivo experiments showed that apoptosis in podocytes was increased in streptozocin (STZ)-induced diabetic rats compared with control rats and that this apoptosis was accompanied by increased macrophages infiltration in the kidney. Then, we established a co-culture system to study the interaction between macrophages and podocytes in the absence or presence of high glucose. Macrophages did not trigger podocytes apoptosis when they were co-cultured in the absence of high glucose in a transwell co-culture system. Additionally, although podocyte apoptosis was increased after high glucose stimulation, there was a further enhancement of podocyte apoptosis when podocytes were co-cultured with macrophages in the presence of high glucose compared with podocytes cultured alone in high glucose. Mechanistically, we found that macrophages were activated when they were exposed to high glucose, displaying pro-inflammatory M1 polarization. Furthermore, conditioned media (CM) from such high glucose-activated M1 macrophages (HG-CM) trigged podocytes apoptosis in a reactive oxygen species (ROS)-p38mitogen-activated protein kinases (p38MAPK) dependent manner, which was abolished by either a ROS inhibitor (Tempo) or a p38MAPK inhibitor (SB203580). Finally, we identified tumor necrosis factor (TNF-a) as a key mediator of high glucose-activated macrophages to induce podocytes apoptosis because an anti-TNF-a neutralizing antibody blunted the apoptotic response, excess ROS generation and p38MPAK activation in podocytes induced by HG-CM. Moreover, addition of recombinant TNF-a similarly resulted in podocytes apoptosis. In summary, the TNF-a that was released by high glucoseactivated macrophages promoted podocytes apoptosis via ROS-p38MAPK pathway. Blockade of TNF-a secretion from high glucose activated macrophages and ROSp38MAPK pathway might be effective therapeutic options to limit podocytes apoptosis and delay the progression of diabetic nephropathy. 


\section{INTRODUCTION}

Podocytes are considered critical cells in the development of diabetic nephropathy (DN) [1]. Reduction in podocytes number mediated by apoptosis has been observed in patients with both early and late DN [2-3] and in animal DN models [4]. The etiology of progressive disorder in podocytes is complex and multifactorial. Studies have suggested that macrophages played an important pathogenic role in this process [5].

Macrophage infiltration is an early response to renal damage, which precedes the decline of renal function and the onset of glomerular damage [6]. The number of macrophage infiltration is closely associated with the severity of proteinuria and histological damage in DN [7]. Glomerular macrophage depletion by diphtheria toxin has been shown to ameliorate podocytes injury in STZ-induced diabetic mouse model [8], thus further supporting a cell-cell interaction between macrophages and podocytes in diabetes mellitus. Recent studies indicated that macrophages are divided into M1 (classically activated macrophages) or M2 (alternatively activated macrophages) subtypes. M1 is characterized by excessive production of inducible nitric oxide synthase (iNOS), TNF- $\alpha$ and interleukin-1 (IL-1), whlie M2 exhibits increased mannose receptor (MR), arginase and IL-10 [9]. We and others have indicated that macrophages in the diabetic kidney were predominantly M1 phenotypes [10-11]. In addition, several studies have demonstrated that M1 macrophages rather than M2 macrophages destroy podocyte integrity and increase its permeability [8]. However, the effect of macrophages on podocytes apoptosis and the molecular mechanisms by which macrophages modulate the apoptosis response in podocytes remain poorly elucidated.

Reactive oxygen species (ROS) are crucial to the initiation of apoptosis in podocytes [12-13]. Previous studies have confirmed that ROS are over-expressed in podocytes in diabetic kidney diseases [14]. P38MAPK (p38mitogen-activated protein kinases), a pro-apoptotic signaling downstream of ROS, is subsequently activated, leading to cell apoptosis [15]. In addition, studies searching for the origin of increased ROS found that TNF- $\alpha$, an effector molecule released by infiltrating macrophages, appears to be central for inducing ROS generation [16]. Therefore, we hypothesized that 1) infiltrating macrophages might promote podocytes apoptosis in DN and 2) mechanistically, TNF- $\alpha$, a key mediator of macrophages, might account for the apoptotic response in podocytes via ROS-p38MAPK pathway.

\section{RESULTS}

\section{Podocyte apoptosis in STZ-induced DN rats}

DN rats developed overt diabetes with higher blood glucose concentrations and lower body weights after the
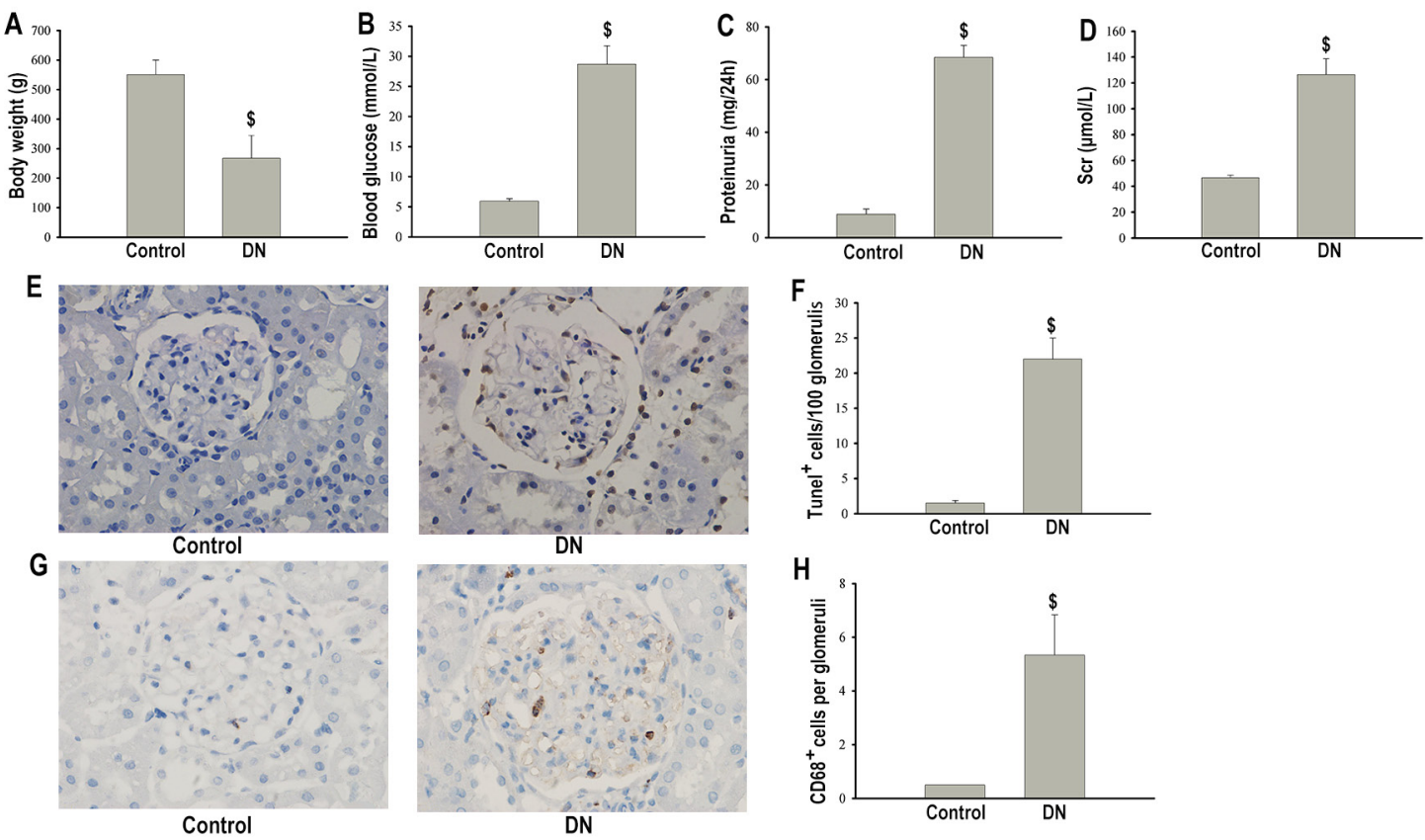

Figure 1: Podocytes apoptosis and macrophage infiltration in nondiabetic or STZ-induced diabetic rats at 18 weeks after STZ injection. Body weight (A). Blood glucose (B). Proteinuria (C). Scr (D). TUNEL staining of apoptotic podocytes $(\times 400)$ (E). Semiquantitation of TUNEL positive cells per 100 glomerulis (F). Immunohistochemical staining of CD68 positive macrophages in glomeruli $(\times 400)(\mathbf{G})$. Quantification of number of CD68 positive macrophages per glomeruli $(\mathbf{H})$. Data were presented as mean \pm SD $(\mathrm{n}=8) .{ }^{\mathrm{S}} \mathrm{P}<0.05$ vs. control group. 
injection of STZ (Figure 1A, 1B). In addition, 24h-urinary proteinuria and serum creatinine (Scr) were markedly increased in diabetic rats than in non-diabetic rats (Figure 1C, 1D). Apoptosis in podocytes detected by TUNEL was significantly increased in the diabetic group compared with the non-diabetic group (Figure 1E, 1F).

\section{Macrophage infiltration in STZ-induced DN rats}

Since inflammatory process has been proposed to contribute to the pathogenesis of DN, we assessed the degree of macrophage infiltration in diabetic glomeruli. Immumohistochemical staining showed that CD68 positive macrophages were markedly increased in the glomeruli of diabetic rats compared with non-diabetic controls (Figure $1 \mathrm{G}, 1 \mathrm{H}$ ).

\section{Macrophagespromoted podocytes apoptosis in the condition of high glucose}

The presence of macrophages in the injured kidney together with the increased apoptotic podocytes led us to examine the possibility that macrophages might promote the death of podocytes. Therefore, we cocultured podocytes with macrophages in a transwell co-culture system in the absence or presence of high glucose, mimicking the diabetic microenvironment in vivo, and examined podocytes apoptosis. Interestingly, we found that macrophages did not trigger podocytes apoptosis when they were co-cultured in the absence of high glucose. Additionally, although both podocytes apoptosis and the apoptotic protein expression of cleaved caspase 3 were increased after high glucose stimulation, there was a further enhancement of podocytes apoptosis and cleaved caspase 3 expression when podocytes were co-cultured with macrophages in the presence of high glucose at podocytes $(\mathrm{P})$ : macrophages $(\mathrm{M})$ ratios of $2: 2$ and 2:4 compared with podocytes cultured in high glucose alone (Figure 2). On the basis of the above results, we speculated that macrophages might be activated by high glucose and then promote podocytes apoptosis.

\section{Macrophages displayed pro-inflammatory M1 activation under high glucose condition}

Next, we detected macrophages phenotypes in the status of high glucose in vitro. The classical marker of M1 (iNOS) and specific marker of M2 macrophages (MR) [9] were measured. After stimulation with high glucose, macrophages increased their expression of iNOS. Additionally, MR was decreased when macrophages were exposed to high glucose compared with those exposed to normal glucose (Figure 3). These data indicated that macrophages displayed pro-inflammatory M1 activation in the condition of high glucose.

\section{Conditioned media from high glucose-activated macrophages (HG-CM) promoted podocytes apoptosis}

As high glucose activated pro-inflammatory M1 macrophages, it was of interest to determine whether such M1 macrophages were uniquely capable of promoting podocytes apoptosis. We collected $\mathrm{CM}$ from normal glucose-cultured macrophages (NC-CM) and from high glucose-activated macrophages (HG-CM) and then applied these media to podocytes. Meanwhile, podocytes treated with normal PRMI 1640 media were used as control. We found that there was no difference in podocytes apoptosis between control group and $\mathrm{NC}-\mathrm{CM}$ group as showed by Annexin V-FITC/PI staining and Hoechst-33342 staining. Apoptosis in podocytes was significantly increased in a time-dependent manner when podocytes were exposed to HG-CM compared with either control or NC-CM (Figure 4), suggesting that such high glucose-activated macrophages directly promoted podocytes apoptosis by releasing a factor or factors.

\section{Apoptosis in podocytes triggered by high glucose-activated macrophages was ROS dependent}

Next, we investigated the mechanisms through which high glucose-activated macrophages induced podocytes apoptosis. Increased ROS has been reported to contribute to podocytes apoptosis in the progression of DN [17]. We then investigated whether ROS was involved in macrophages mediated podocytes apoptosis. We measured ROS level in podocytes cultured with HG-CM by DCFHDA analysis. There was no difference in ROS generation between the control group and the NC-CM group. However, ROS generation was significantly increased when podocytes were exposed to HG-CM (Figure 5A, 5B). Consistent with the results in vitro, 8-hydroxy-2-deoxyguanosine(8-OHdG) immunostaining also showed enhanced ROS in podocytes in STZ-induced diabetic rats (Figure 5E, 5F). Tempo, an inhibitor of ROS, markedly decreased HG-CM mediated ROS generation (Figure 5A, 5B). Moreover, Tempo reduced the proportion of apoptotic podocytes and cleaved caspase 3 expression elevated by HG-CM (Figure 5I-5M), thus validating a role for ROS in mediating the pro-apoptotic effect of high glucose-activated macrophages on podocytes.

\section{Inhibition of p38MAPK blunted podocytes apoptosis induced by high glucose-activated macrophages}

We next tried to identify the molecular link between ROS activation and podocytes apoptosis induced by macrophages. It has been reported that excess ROS promoted cell apoptosis by p38MAPK pathway [15], We investigated whether the p38MAPK pathway was 
involved in high glucose-activated macrophages mediated podocytes apoptosis. The p38MAPK activation detected by the ratio of phosphorylated p38MAPK (p-p38MAPK) to total p38MAPK was markedly increased when podocytes were exposed to $\mathrm{HG}-\mathrm{CM}$ compared with the ratios found in the control and $\mathrm{NC}-\mathrm{CM}$ groups (Figure 5C, 5D). Consistent with this finding, p-p38MAPK/p38MAPK in kidney tissue was significantly increased in the diabetic rats compared with the control rats (Figure 5G, 5H). Furthermore, the phosphorylation effect of $\mathrm{HG}-\mathrm{CM}$ on the p38MAPK in podocytes was blocked by either Tempo or a specific p38MAPK inhibitor (SB203580) (Figure 5C, 5D). In addition, SB203580 effectively decreased podocytes apoptosis and cleaved caspase 3 expression induced by HG-CM (Figure 5I-5M). These data suggested a direct link between ROS and p38MAPK, which accounted, at least in part, for the apoptotic response of podocytes triggered by high glucose-activated macrophages.

\section{Macrophage derived TNF- $\alpha$ is required to promote apoptosis in podocytes}

We have demonstrated that macrophages promoted podocytes apoptosis by releasing a factor or factors, which subsequently activated the pro-apoptotic ROSp38MAPK pathway. Studies have demonstrated that TNF- $\alpha$ was a potent inducer of ROS production [18]. In particular, TNF- $\alpha$ derived from macrophages was recently described as a critical pathogenic factor in DN [19]. Therefore, we decided to focus on TNF- $\alpha$. We speculated that macrophages derived TNF- $\alpha$ might be a key fuse for ROS-p38MAPK pathway and subsequent apoptosis in podocytes. First of all, we detected the level of TNF- $\alpha$ in the $\mathrm{CM}$ from macrophages, which was evidently increased in $\mathrm{HG}-\mathrm{CM}$ compared with NC-CM (Figure 6A). Western blot analysis showed that protein expression of TNF- $\alpha$ was also increased when macrophages were exposed to high
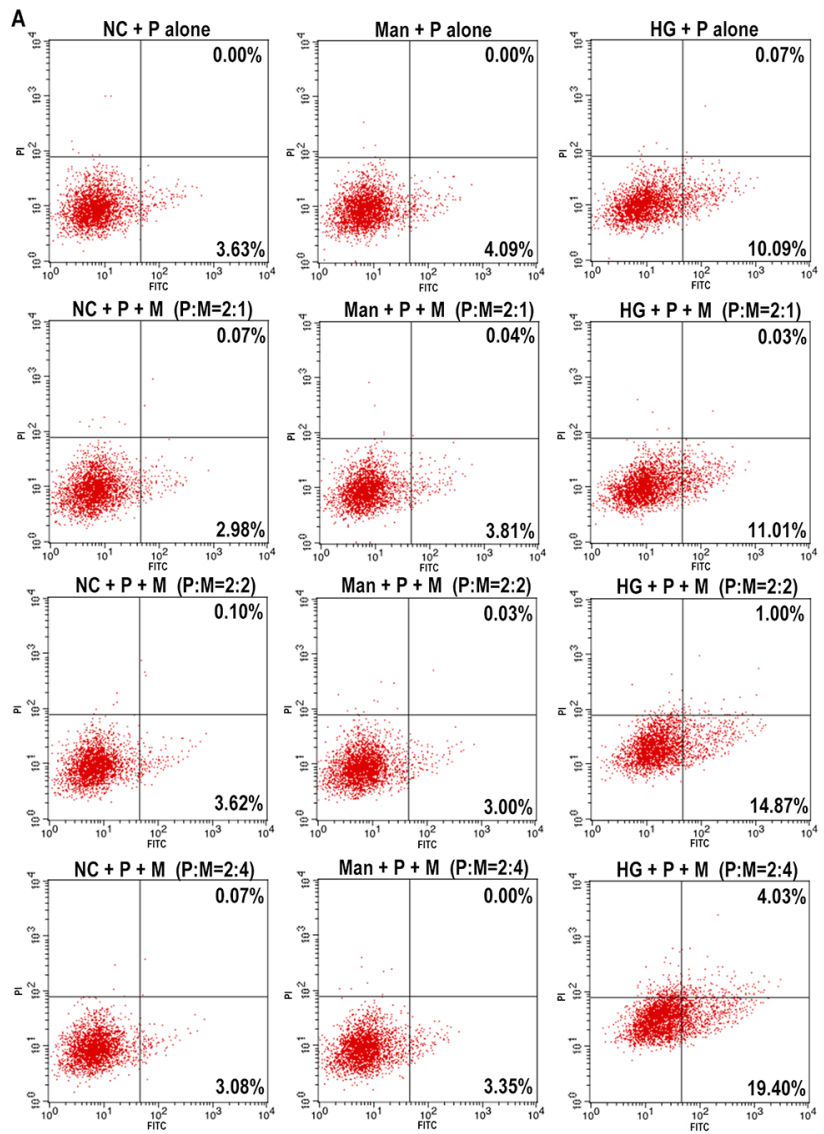

B

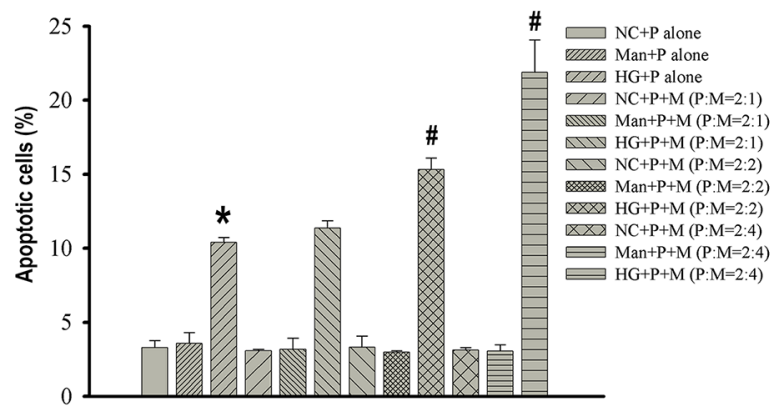

C

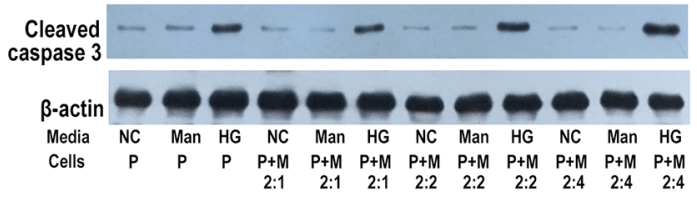

D

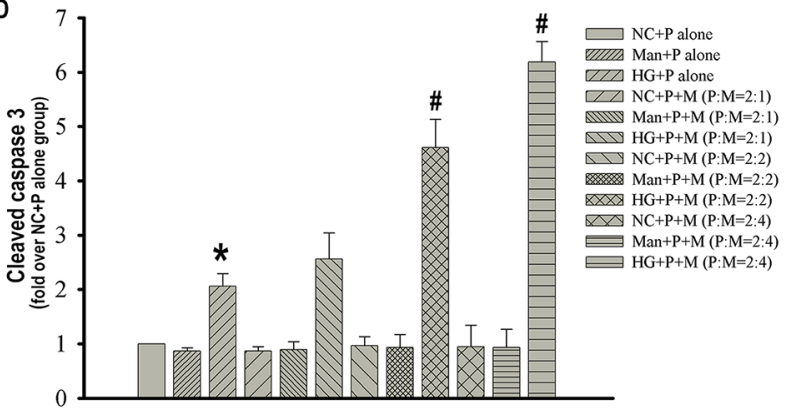

Figure 2: Macrophages promoted podocytes apoptosis in the condition of high glucose. Flow cytometry analysis of podocytes apoptosis with Annexin V-FITC/PI staining (A). Percentage of apoptotic podocytes (B). Representative western blotting analysis of cleaved caspase 3 in podocytes (C). $\beta$-actin was used as an internal control. Quantification of cleaved caspase 3 protein expression (D). NC+P alone: podocytes treated with normal PRMI 1640 media. Man+P alone: Podocytes treated with 25 mM mannitol. HG+P alone: Podocytes treated with $25 \mathrm{mM}$ high glucose. $\mathrm{NC}+\mathrm{P}+\mathrm{M}$ : Transwell co-culture of podocytes $(\mathrm{P})$ and RAW 264.7 cells $(\mathrm{M})$ in the absence of $25 \mathrm{mM}$ high glucose at indicated ratios of $\mathrm{P}$ to $\mathrm{M}$. Man $+\mathrm{P}+\mathrm{M}$ : Transwell co-culture of $\mathrm{P}$ and $\mathrm{M}$ in $25 \mathrm{mM}$ mannitol at indicated ratios of $\mathrm{P}$ to $\mathrm{M}$. $\mathrm{HG}+\mathrm{P}+\mathrm{M}$ : Transwell co-culture of $\mathrm{P}$ and $\mathrm{M}$ in the presence of $25 \mathrm{mM}$ high glucose at indicated ratios of $\mathrm{P}$ to $\mathrm{M}$. Data were presented as mean $\pm \mathrm{SD}$ from three independent experiments. ${ }^{*} \mathrm{P}<0.05$ vs. $\mathrm{NC}+\mathrm{P}$ alone group; ${ }^{\sharp} \mathrm{P}<0.05$ vs. HG $+\mathrm{P}$ alone group. 
glucose compared with those cultured in normal glucose (Figure 6B, 6C). In addition, anti-TNF- $\alpha$ neutralizing antibody blunted the increased ROS production and p-p38MAPK/p38MAPK expression (Figure 6D-6G) and blocked the subsequent apoptotic rate by approximately $60 \%$ and cleaved caspase 3 expression in podocytes elicited by HG-CM (Figure 6H-6L), indicating TNF- $\alpha$ secreted from high glucose-activated macrophages was the essential factor in the above process. To unequivocally determine the function of TNF- $\alpha$ in podocytes apoptosis, we cultured podocytes with TNF- $\alpha$ alone and observed an obvious increase in apoptotic podocytes and cleaved caspase 3 expression (Figure 6H-6L). These data further argued for a pro-apoptotic function of this factor. Moreover, both ROS generation and p-p38MAPK/ p38MAPK expression were increased when podocytes were exposed to TNF- $\alpha$ alone (Figure 6D-6G).

\section{DISCUSSION}

The cell-cell interaction between infiltrating inflammatory cells and renal intrinsic cells has been the subject of extensive investigation in recent years in experimental inflammatory nephropathies, placing emphasis on the cross-talk between macrophages and renal cells such as mesangial cells, tubule cells and renal cell carcinoma cells with respect to cellular events such as proliferation, matrix synthesis, apoptosis and metastasis [20-22]. Our previous studies have demonstrated a profibrotic function of monocytes on proximal tubular epithelial cells (PTEC). Binding of monocytes via the intracellular adhesion molecule-1 (ICAM-1) expressed on the basolateral surface of PTEC stimulated the synthesis of TGF- $\beta$ [23]. As podocytes are critical in maintaining glomerular function [24], several studies recently attempted to evaluate the role of macrophages on podocytes structure and function in DN despite the fact that they infiltrated in the glomeruli with diabetes in a low number, showing that macrophages had the potential to mediate podoctyes impairment [8]. However, the effect of macrophages on podocytes apoptosis remains poorly elucidated. Therefore, it is imperative to understand the cell-cell interaction between macrophages and podoctyes comprehensively and to identify effective therapeutic interventions and strategies to block the development of diabetic kidney disease.

In the current study, we firstly identified a novel correlation of macrophages with podocytes impairment in the progression of DN. Podocyte apoptosis was accompanied by an increased number of infiltrating macrophages in renal tissue in STZ induced diabetic rats. Next, we constructed a transwell co-culture system to characterize the intercellular communication between macrophages and podocytes. In such co-culture system, macrophages exerted a further pro-apoptotic effect on podocytes in the presence of high glucose but not in the absence of high glucose. Moreover, macrophages exhibited pro-inflammatory M1 phenotypes featured by increased cytokines such as iNOS and TNF- $\alpha$ when they were exposed to high glucose. Subsequently, we found that the CM from such high glucose-activated macrophages rather than inactivated macrophages promoted podocytes apoptosis via a panel of soluble molecules. Indeed, the requirement for macrophage activation to induce podocytes injury is consistent with other studies in which whether adoptively transferred macrophages
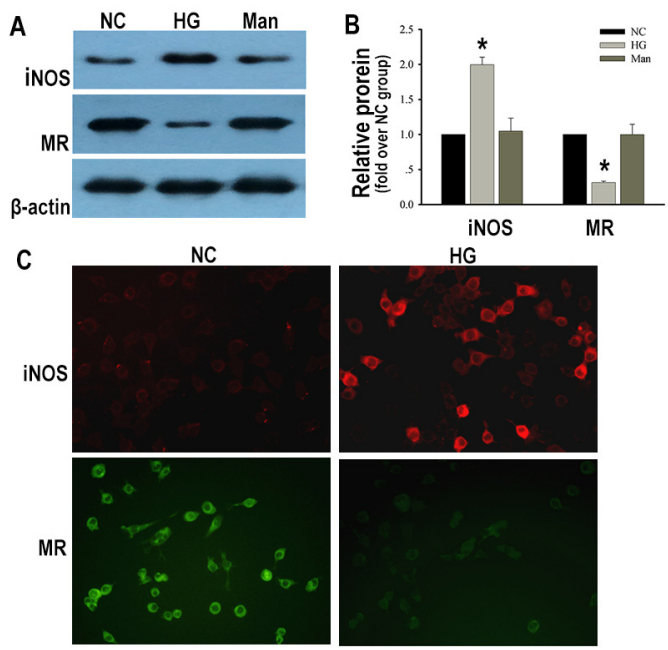

Figure 3: Macrophages displayed pro-inflammatory M1 activation under high glucose condition. Representative western blotting analysis of iNOS and MR in macrophages (A). Quantification of iNOS and MR protein expression in macrophages (B). Immunofluorescent staining of iNOS and MR in macrophages $(\times 200)(\mathbf{C})$. NC: RAW 264.7 cells treated with normal glucose for $24 \mathrm{~h}$. HG: RAW 264.7 cells treated with $25 \mathrm{mM}$ high glucose for $24 \mathrm{~h}$. Man: RAW 264.7 cells treated with $25 \mathrm{mM}$ mannitol for $24 \mathrm{~h}$. Data were presented as mean $\pm \mathrm{SD}$ from three independent experiments. ${ }^{*} \mathrm{P}<0.05$ vs. NC group. 
could cause renal injury is dependent on their activation status [25]. Herein, we provided direct evidence of the pathogenic effect of high glucose-activated macrophages on podocytes apoptosis and loss in diabetic nephropathy.

How did high glucose-activated macrophages lead to podocytes apoptosis? Recent studies have painted an interesting picture on the emerging role of oxidative stress, whereby the excessive generation of ROS is associated with the structural and functional damage of various organs. Then, several intracellular transduction signal pathways are activated in response to oxidative stress, and regulate cell senescence, proliferation and apoptosis [15]. Data from animal and cell studies showed elevated levels of ROS accumulation in diabetic kidneys and renal parenchyma cells under multiple stimulus $[15,26]$. Susztak et al. reported that the increased ROS generation was a potential mediator of podocytes apoptosis in DN. Mechanistically, elevated ROS induced podocytes apoptosis via the activation of pro-apoptotic
p38MAPK pathway [4], which was also required for the induction of apoptosis by TGF- $\beta$ in podocytes [27]. In the present study, we observed an increase of both ROS generation and its downstream signal molecule p-p38MAPK activation when podocytes were exposed to HG-CM but not NC-CM. In addition, podocyte apoptosis triggered by HG-CM was markedly reduced by an ROS inhibitor (Tempo) or a p38MAPK inhibitor (SB203580). These results indicated that high glucose-activated macrophages led podocytes to an apoptotic response via ROS-p38MAPK pathway. Moreover, we provided a new relationship between inflammation and oxidative stress that macrophages derived soluble molecules constituted another trigger for ROS generation in podocytes that are responsible for cells apoptosis and the progression of DN.

Activated macrophages secreting pro-inflammatory mediators is a vital event in the initiation of DN. TNF- $\alpha$ is increasingly considered as a potential mediator of macrophages and contributes to renal injury. Many animal
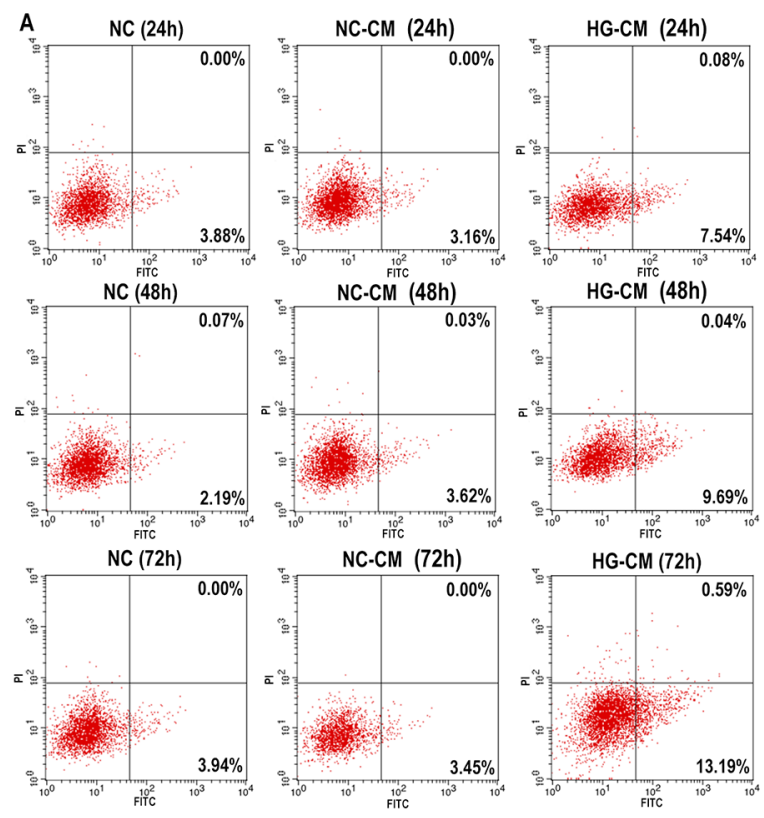

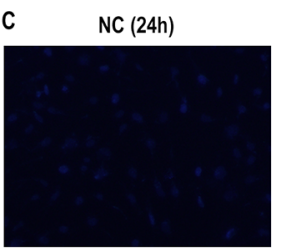

NC (48h)
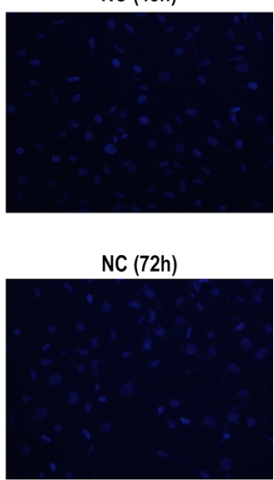

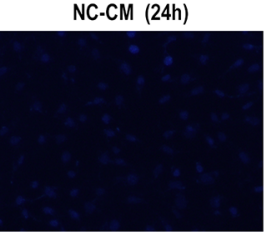

NC-CM $(48 \mathrm{~h})$

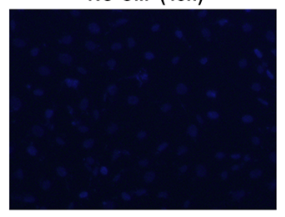

NC-CM $(72 \mathrm{~h})$

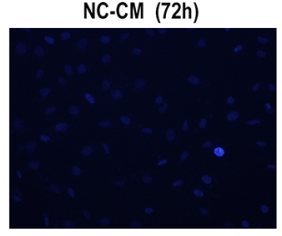

HG-CM (24h)

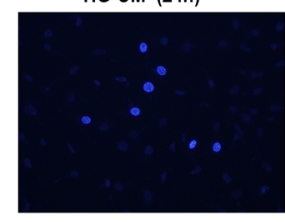

HG-CM (48h)

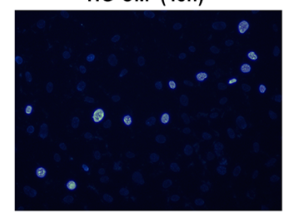

HG-CM (72h)

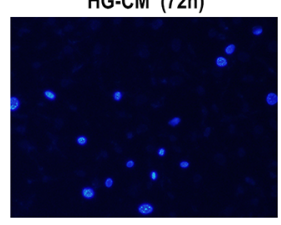

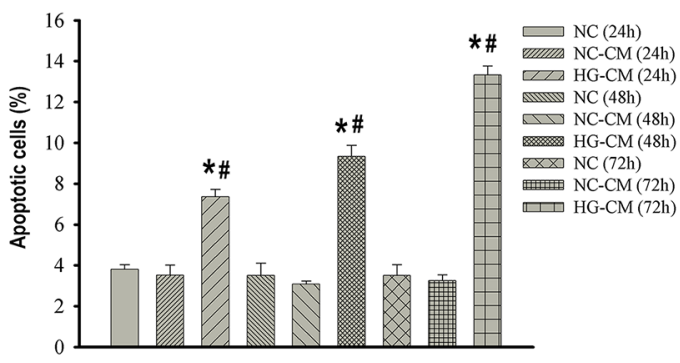

Figure 4: Conditioned media from high glucose-activated macrophages (HG-CM) promoted podocytes apoptosis. Flow cytometry analysis of podocytes apoptosis with Annexin V-FITC/PI staining (A). Percentage of apoptotic podocytes (B). Hoechst-33342 staining of apoptotic podocytes $(\times 200)($ C). NC: podocytes treated with norml RPMI 1640 media for indicated time. NC-CM: podocytes treated with $\mathrm{NC}-\mathrm{CM}$ for indicated time. $\mathrm{HG}-\mathrm{CM}$ : podocytes treated with $\mathrm{HG}-\mathrm{CM}$ for indicated time. Data were presented as mean $\pm \mathrm{SD}$ from three independent experiments. ${ }^{*} \mathrm{P}<0.05$ vs. $\mathrm{NC}$ group; ${ }^{\mathrm{P}} \mathrm{P}<0.05$ vs. $\mathrm{NC}-\mathrm{CM}$ group at the same time point, respectively. 
and clinical studies have reported that TNF- $\alpha$ expression was increased in the urinary and diabetic kidney, which occureed prior to the rise in albuminuria and was independently correlated with clinical markers of glomerular and tubulointerstitial injury [28-29]. Inhibition of TNF- $\alpha$ with a neutralizing antibody reduced the features
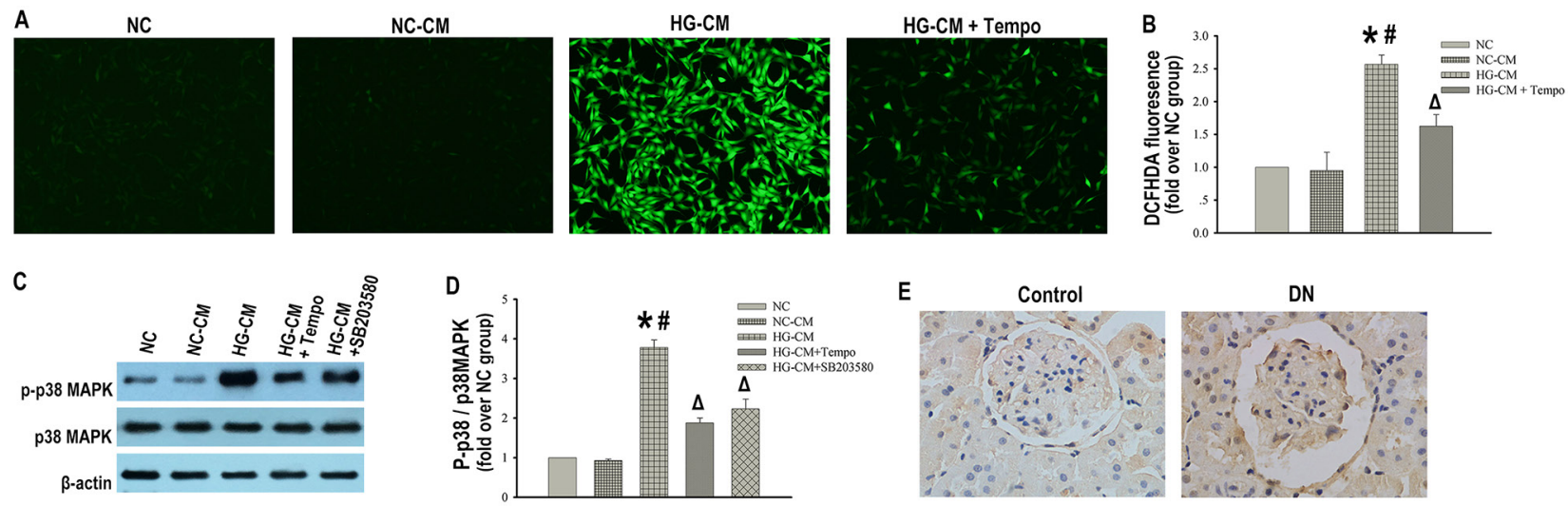

E
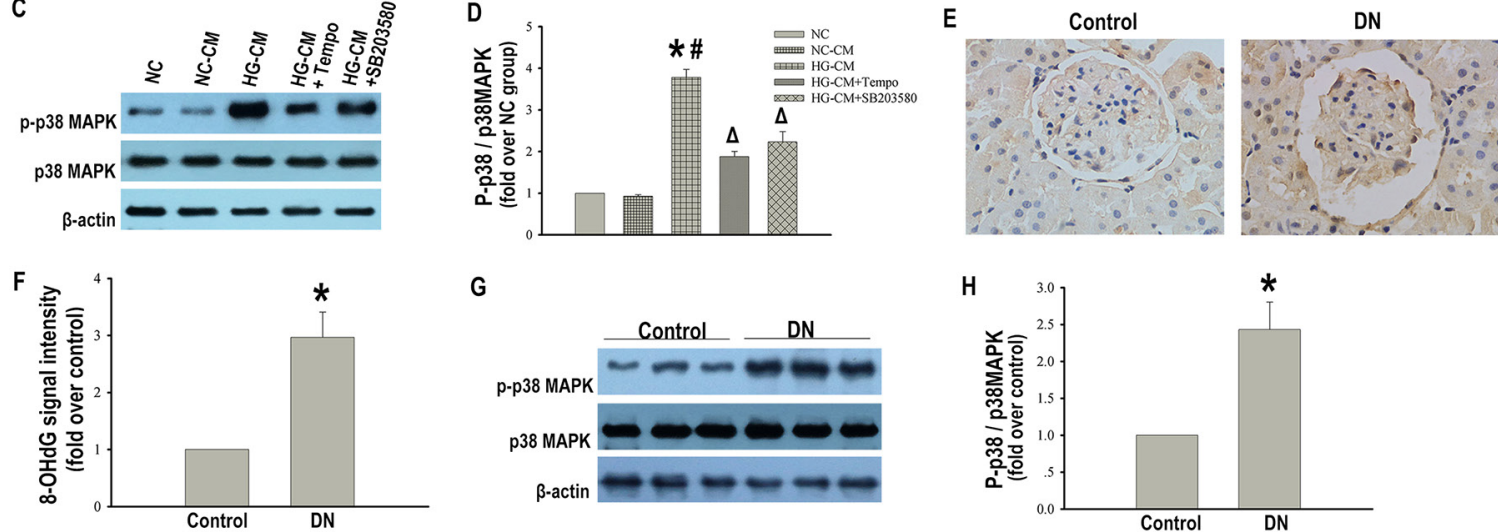

G
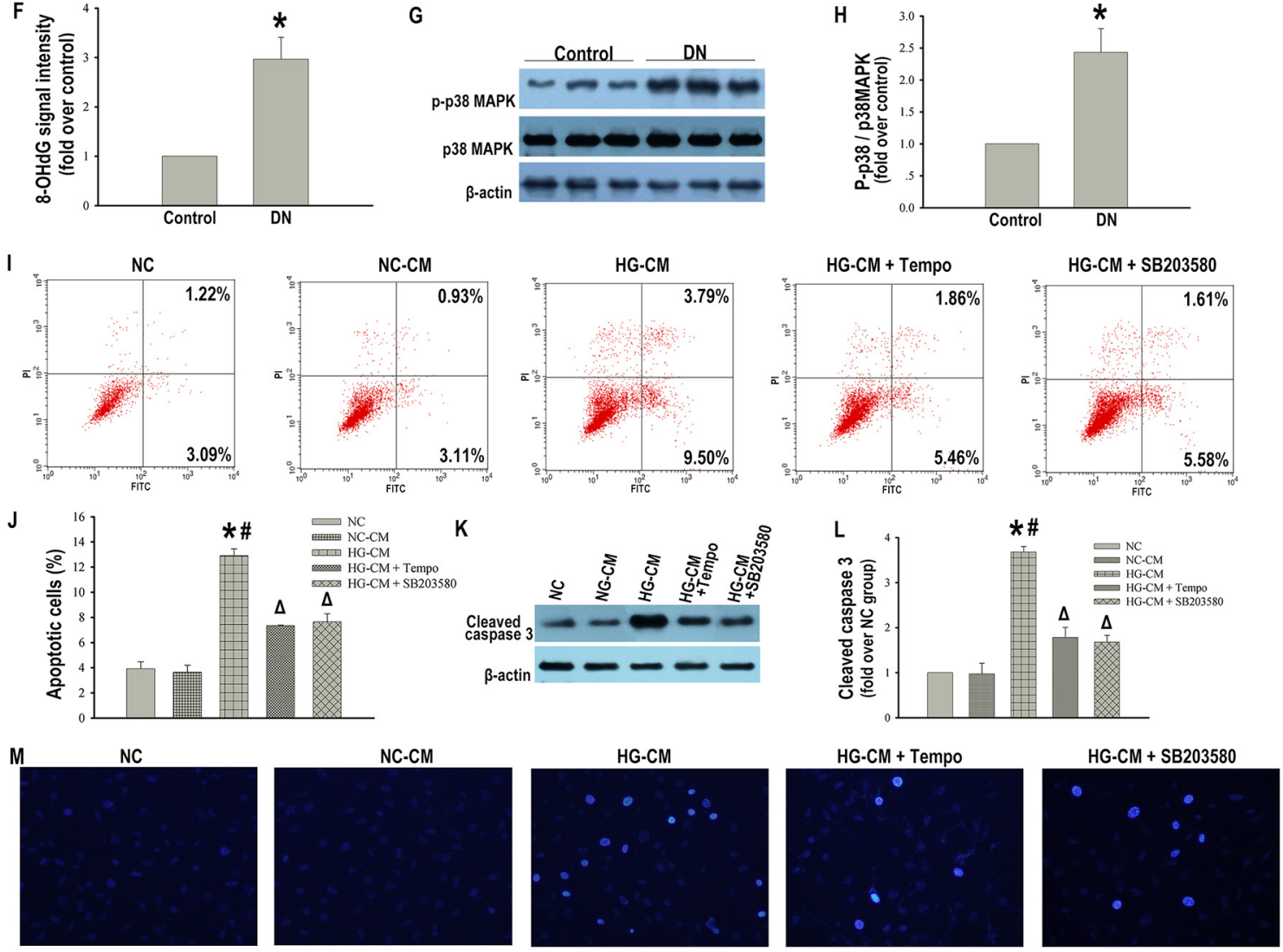

Figure 5: Apoptosis in podocytes triggered by high glucose-activated macrophages was ROS-p38MAPK dependent. Representative DCFHDA staining of ROS in podocytes $(\times 100) \mathbf{( A )}$. Quantitative analysis of ROS (B). Representative western blotting analysis of p-p38MAPK and p38MAPK in podocytes (C). $\beta$-actin was used as an internal control. Quantification of p-p38MAPK/p38MAPK in podocytes (D). Immunohistochemical staining of $8-\mathrm{OHdG}$ in glomerulus $(\times 400)$ of control or diabetic rats $(\mathbf{E})$. Semiquantitative analysis of 8-OHdG signal intensity (F). Representative western blotting analysis of p-p38MAPK and p38MAPK in glomerular lysates of control or diabetic rats (G). Quantification of p-p38MAPK/p38MAPK in glomerular lysates $(\mathbf{H})$. Data were presented as mean $\pm \mathrm{SD}(\mathrm{n}=8)$. $* \mathrm{P}<0.05$ vs. control group. Flow cytometry analysis of podocytes apoptosis with Annexin V-FITC/PI staining (I). Percentage of apoptotic podocytes (J). Representative western blotting analysis of cleaved caspase 3 in podocytes (K). Quantification of cleaved caspase 3 protein expression in podocytes (L). Hoechst-33342 staining of apoptotic podocytes $(\times 200)(\mathbf{M})$. NC: podocytes treated with norml RPMI 1640 media. NCCM: podocytes treated with NC-CM. HG-CM: podocytes treated with HG-CM. HG-CM + Tempo: podocytes treated with HG-CM and a ROS inhibitor (Tempo). HG-CM + SB203580: podocytes treated with HG-CM and a MAPK inhibitor (SB203580). Data were presented as

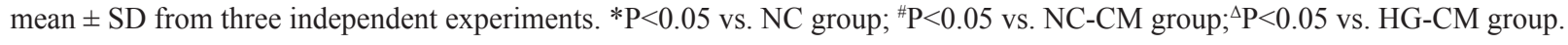


of DN in both type 1 and type 2 diabetes mellitus [19, 30]. However, TNF- $\alpha$ is produced not only by macrophages, but also by various renal resident cells such as mesangial cells and podocytes [31-32]. Therefore, the source of TNF- $\alpha$ that drives podocytes apoptosis in diabetic nephropathy remains unknown. Awad et al. demonstrated that

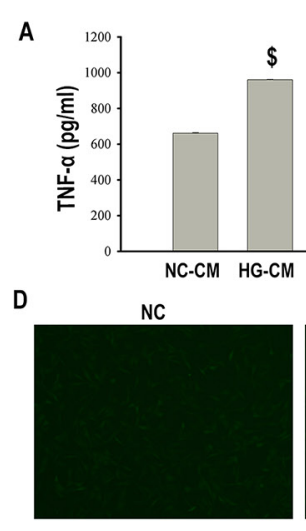

E

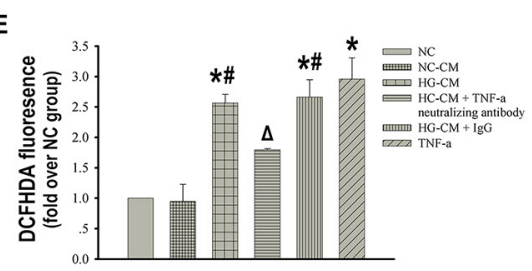

H

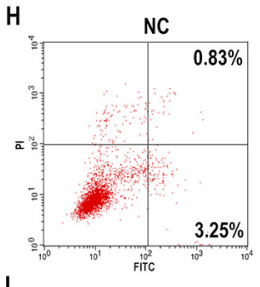

I

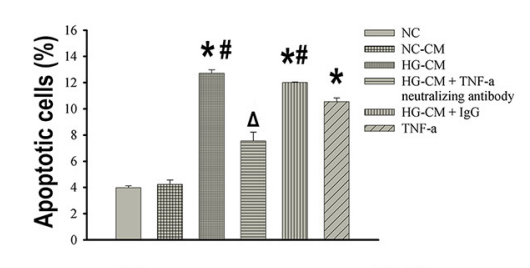

L

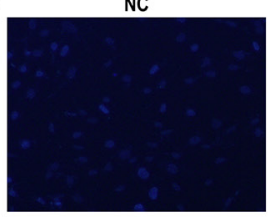

NC-CM

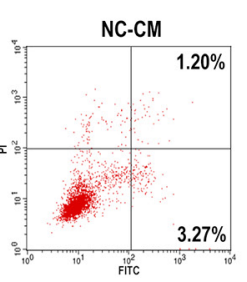

NC-CM

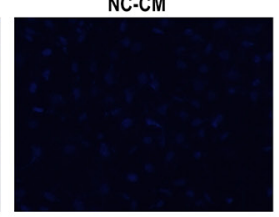

B

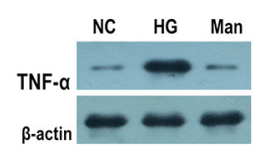

C
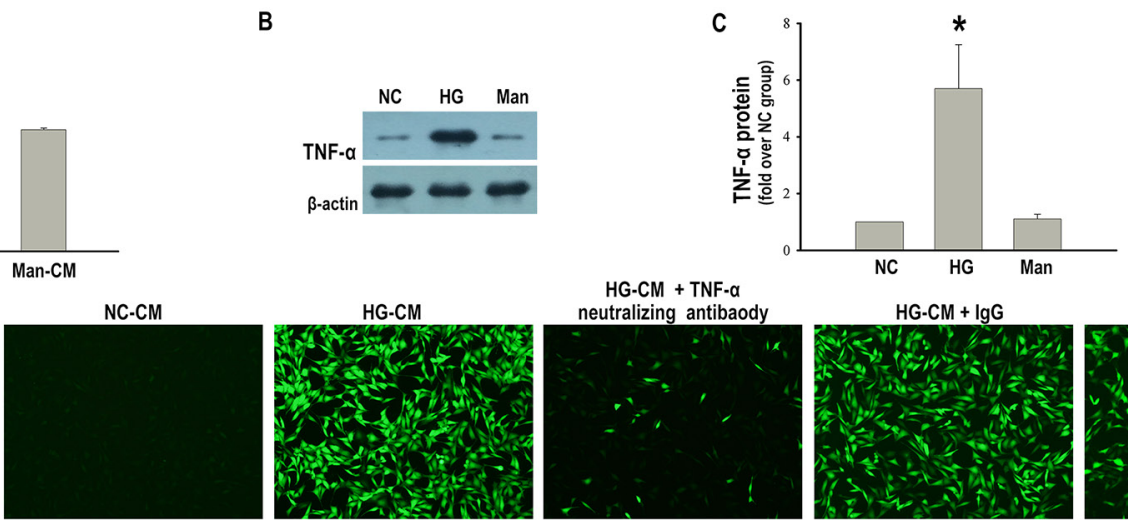

F

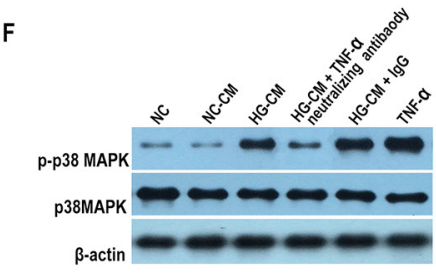

HG-CM + TNF- $a$

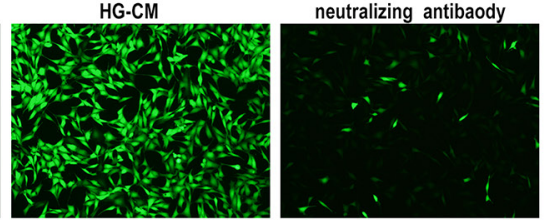

G
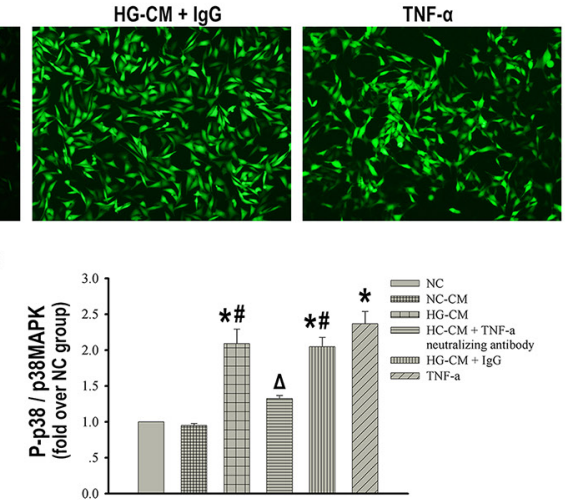

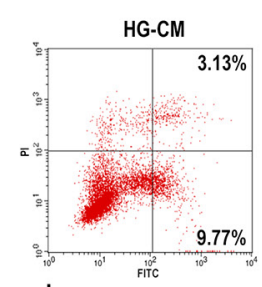

$\mathrm{J}$
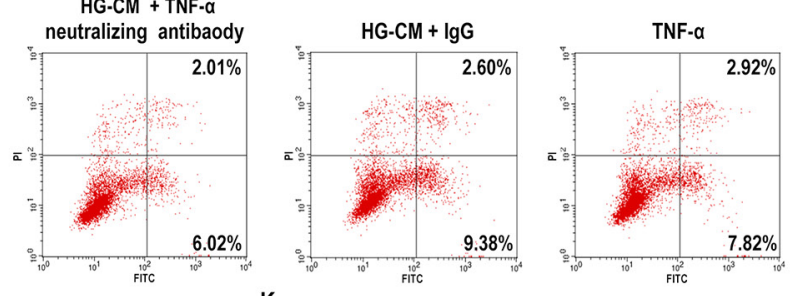

K
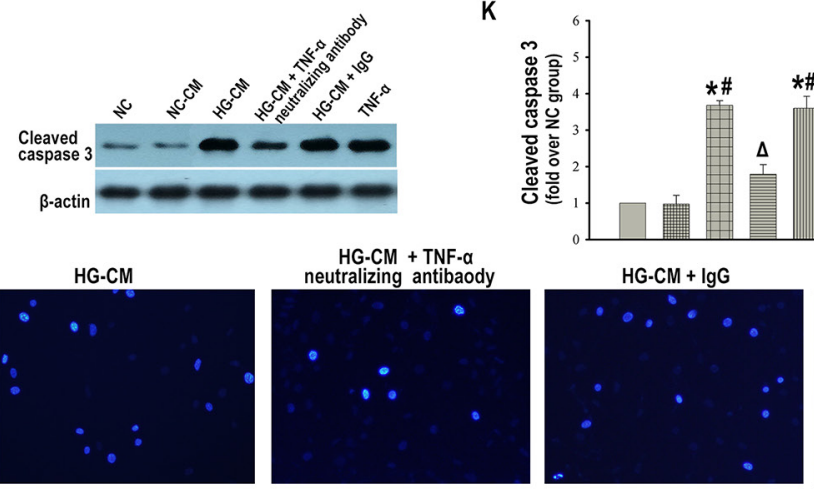

* $\begin{gathered}\text { NC } \\ \text { NC-CM } \\ \text { HG-CM }\end{gathered}$

HG-CM + TNF-
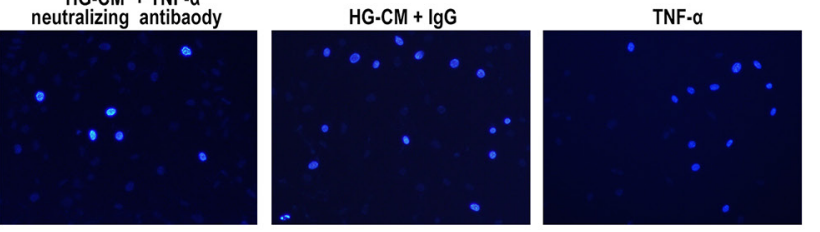

Figure 6: Macrophages derived TNF- $\alpha$ is required to promote apoptosis in podocytes. ELISA assay of TNF- $\alpha$ level in the culture supernatant of macrophages (A). Representative western blotting analysis of TNF- $\alpha$ in macrophages (B). $\beta$-actin was used as an internal control. Quantification of TNF- $\alpha$ in macrophages (C). NC-CM: CM from normal glucose-treated RAW 264.7 cells. HGCM: CM from 25 mM high glucose-treated RAW 264.7 cells. Man-CM: CM from 25 mM mannitol-treated RAW 264.7 cells. NC: RAW 264.7 cells treated with normal glucose. HG: RAW 264.7 cells treated with $25 \mathrm{mM}$ high glucose. Man: RAW 264.7 cells treated with 25 $\mathrm{mM}$ mannitol. Data were presented as mean $\pm \mathrm{SD}$ from three independent experiments. ${ }^{\$} \mathrm{P}<0.05$ vs. NC-CM group; $* \mathrm{P}<0.05$ vs. NC group. Representative DCFHDA staining of ROS $(\times 100)$ (D). Quantitative analysis of ROS (E). Representative western blotting analysis of p-p38MAPK and p38MAPK in podocytes (F). Quantification of p-p38MAPK/p38MAPK in podocytes (G). Flow cytometry analysis of podocytes apoptosis with Annexin V-FITC/PI staining (H). Percentage of apoptotic podocytes (I). Representative western blotting analysis of cleaved caspase 3 in podocytes $(\mathbf{J})$. Quantification of cleaved caspase 3 protein expression in podocytes (K). Hoechst-33342 staining of apoptotic podocytes $(\times 200)(\mathbf{L})$. NC: podocytes treated with norml RPMI 1640 media. NC-CM: podocytes treated with NC-CM. HG-CM: podocytes treated with HG-CM. HG-CM + TNF- $\alpha$ neutralizing antibody: podocytes treated with HG-CM and anti-TNF- $\alpha$ neutralizing antibody. HG-CM + IgG: podocytes treated with HG-CM and IgG1. TNF- $\alpha$ : podocytes treated with TNF- $\alpha$. Data were presented as mean $\pm \mathrm{SD}$ from three independent experiments. ${ }^{*} \mathrm{P}<0.05$ vs. NC group; ${ }^{\#} \mathrm{P}<0.05$ vs. NC-CM group; ${ }^{\wedge} \mathrm{P}<0.05$ vs. HG-CM group. 
specific deletion of TNF- $\alpha$ in macrophages significantly blocked both the basic and diabetes induced TNF- $\alpha$ levels and reduced albuminuria, plasma creatinine and histopathologic changes in diabetic animals [19]. Another study supporting a detrimental effect of macrophages derived TNF- $\alpha$ on cultured podocytes showed that this cytokine released by macrophages induced cytoskeleton reorganization [33]. In the present study, we found that the level of TNF- $\alpha$ was increased in the HG-CM. Anti-TNF- $\alpha$ neutralizing antibody abrogated podocyte apoptosis, excess ROS generation and $\mathrm{p} 38 \mathrm{MPAK}$ activation elicited by HG-CM. Moreover, addition of TNF- $\alpha$ alone mimicked the pro-apoptotic effect of HG-CM on podocytes. Taken together, these data indicated that TNF- $\alpha$ released by high glucose-activated macrophages mediated podocytes apoptosis via TNF- $\alpha$-ROS-p38MAPK pathway. However, an anti-TNF- $\alpha$ neutralizing antibody did not completely abolish ROS production, p38MPAK activation and subsequent apoptosis in podocytes, which suggests that other facts might also compensate for the apoptotic response in podocytes induced by high glucose-activated macrophages. Studies designed to identify these unknown mediators would provide more clues for understanding the exact role of macrophages in podocytes impairment in diabetic kidney disease.

The current study demonstrated that TNF- $\alpha$ secreted by infiltrating macrophages in diabetic kidney mediated a novel ROS-p38MPAK dependent apoptosis in podocytes. Blockade of TNF- $\alpha$ secretion from high glucose activated macrophages and ROS-p38MAPK pathway might be effective therapeutic strategies for limiting podocytes apoptosis and the progression of diabetic nephropathy.

\section{MATERIALS AND METHODS}

\section{Animal experiments}

The experimental protocol was approved by the Ethical Committee of Southeast University. Healthy male Sprague-Dawley rats weighing 200-220g were purchased from Shanghai Slac Laboratory Animal Co.Ltd (Shanghai, China). Rats were maintained under standard room temperature and regular $12 \mathrm{~h}$ photoperiods, and were allowed free access to food and water. After one week of acclimatization, rats were randomly divided into two groups: (1) Control ( $n=8)$; (2) DN ( $n=8)$. DN was induced with a single intraperitoneal injection of streptozotocin (STZ, sigma) dissolved in $0.1 \mathrm{M}$ citrate buffer $(\mathrm{pH} 4.5)$ at $58 \mathrm{mg} / \mathrm{kg}$, while control rats received $0.1 \mathrm{M}$ citrate buffer solution. Three days later, diabetic rats exhibited elevated blood glucose levels ( $\geq 16.7 \mathrm{mmol} / \mathrm{L})$ by measuring tail blood glucose (BG) level. All animals were weighed and sacrificed at 18 weeks after diabetes was induced. Before being sacrificed, an individual rat was placed in metabolic cages to obtain $24 \mathrm{~h}$ urine. Blood samples were collected for biochemical studies, and kidneys were collected for histological examination and molecular assays.

\section{Macrophages culture}

The mouse macrophage cells (RAW 264.7) were purchased from Shanghai Institutes for Biological Sciences (Shanghai, China). Cells were routinely maintained in normal RPMI 1640 media containing $11.1 \mathrm{mM}$ glucose supplemented with $10 \%$ fetal bovine serum (FBS), $100 \mathrm{U} / \mathrm{ml}$ penicillin and $100 \mu \mathrm{g} / \mathrm{ml}$ streptomycin, and incubated at $37^{\circ} \mathrm{C}$ in $5 \%$ $\mathrm{CO} 2$. Conditioned media (CM) was prepared as described previously [33]. In brief, RAW 264.7 cells were seeded on six well-tissue culture plates, and incubated with or without $25 \mathrm{mM}$ high glucose for $24 \mathrm{~h}$. The $25 \mathrm{mM}$ high glucose was achieved by adding $13.9 \mathrm{mM}$ glucose to normal RPMI 1640 media (containing $11.1 \mathrm{mM}$ glucose). Then, the cells were washed three times to remove high glucose and debris, and cultured in normal RPMI 1640 media for a further $24 \mathrm{~h}$. Then, these supernatants were collected, centrifuged at 2000rpm for 10 min and referred to as CM from normal glucose-cultured macrophages (NC-CM) or CM from $25 \mathrm{mM}$ high glucoseactivated macrophages (HG-CM), respectively.

\section{Podocytes culture}

The mouse conditionally immortalized podocyte cell line (kindly provided by Peter Mundel at the Mount Sinai School of Medicine, USA) between passage 14 and 20 was cultured on dishes coated with type I collagen (BD, USA) and in normal RPMI 1640 media supplemented with $10 \% \mathrm{FBS}, 100 \mathrm{U} / \mathrm{ml}$ penicillin, and $100 \mathrm{U} / \mathrm{ml}$ streptomycin at $33^{\circ} \mathrm{C}$ in the presence of $10 \mathrm{U} / \mathrm{ml}$ mouse recombinant interferon- $\gamma$ (Sigma, USA) and then differentiated for 1014 days at $37^{\circ} \mathrm{C}$ in the absence of interferon- $\gamma$.

\section{Co-culture macrophages with podocytes}

In a transwell co-culture system, RAW 264.7 cells $\left(2 \times 10^{5}, 4 \times 10^{5}, 8 \times 10^{5}\right)$ seeded on a $0.4 \mu \mathrm{m}$ Transwell insert (Millipore) were co-cultured with podocytes $\left(4 \times 10^{5}\right)$ for $48 \mathrm{~h}$ in the absence or presence of $25 \mathrm{mM}$ high glucose treatment. The ratios of podocytes $(\mathrm{P})$ to macrophages $(\mathrm{M})$ were $2: 1,2: 2$ and $2: 4$, respectively.

In the $\mathrm{CM}$ experiments, podocytes $\left(4 \times 10^{5}\right)$ planted on six well plates were cultured overnight in normal RPMI 1640 media. Then, the cells were washed with PBS three times. After that, normal PRMI 1640 media (control), $\mathrm{NC}-\mathrm{CM}$ or HG-CMwas added to podocytes for $24-72 \mathrm{~h}$. In some experiments, $10 \mu \mathrm{g} / \mathrm{ml}$ anti-TNF- $\alpha$ neutralizing antibody (RD, USA), $10 \mu \mathrm{g} / \mathrm{ml}$ IgG1 Isotype control (RD, USA), 300 $\mu \mathrm{M}$ ROS inhibitors (Tempo, sigma) or $10 \mu \mathrm{M}$ p38MAPK inhibitor (SB203580, RD, USA) was respectively added to cells with CM for $72 \mathrm{~h}$. Besides, $10 \mathrm{ng} / \mathrm{ml}$ recombinant mouse TNF- $\alpha$ (RD, USA) alone was applied to incubate podocytes for $72 \mathrm{~h}$ when necessary.

\section{Urine chemistry analysis}

Scr was analyzed by an automatic biochemistry analyzer (Hitachi, Japan). 24 h-urinary proteinuria was 
measured using an ELISA Kit (Jian Cheng, China) according to the manufacturer's method.

\section{Immunohistochemical staining}

Immunohistochemistry was performed on paraffin sections using a microwave based antigen retrieval technique. Sections were incubated with primary CD68 antibody (Santa Cruz, USA), 8-hydroxy-2-deoxyguanosine (8-OHdG, Santa, USA) antibody followed by appropriate secondary antibodies incubation. The immunostaining was visualized using $\mathrm{DAB}$, and the slides were counterstained with hematoxylin. The mean number of $\mathrm{CD} 68$ positive macrophages was evaluated by counting in twenty randomly selected glomeruli. An immunohistochemistry semiquantitative analysis of 8-OHdG signal intensity was evaluated in twenty randomly selected areas using the Image-Pro Plus image analysis system.

\section{Measurement of apoptosis}

A terminal deoxynucleotidyl transferase-mediated dUTP nick-end labeling (TUNEL) assay with an In Situ Cell Death Detection Kit (Roche, Germany) was performed to determine apoptosis within glomeruli following the manufacturer's protocol. Briefly, kidney tissues were fixed in $4 \%$ paraformaldehyde, incubated with TUNEL reaction mixture and then followed by antiFluorescein-POD conjugate. The degree of apoptosis was estimated using a scale based on the mean number of TUNEL-positive cells per 100 glomerular sections.

Annexin V-FITC/PI (BD, USA) staining was used to identify apoptotic cells in vitro according to the manufacturer's instructions. After being cultured with certain stimulation, cells were harvested, and centrifuged at $1200 \mathrm{rpm} / \mathrm{min}$ for $5 \mathrm{~min}$, rinsed with PBS twice and resuspended in $400 \mu \mathrm{l} 1 \times$ binding buffer containing $5 \mu \mathrm{l}$ PI and $5 \mu \mathrm{l} \mathrm{V-FITC,} \mathrm{incubated} \mathrm{for} 15 \mathrm{~min}$ at the room temperature in the dark. The cell suspension was determined by flow cytometry to analyze the apoptotic rate. Cells in the upper-right quadrant and lower-right quadrant were classified as apoptotic.

Hoechst-33342 (Beyotime, China) staining was also used to evaluate podocyte apoptosis in vitro. After being fixed with $4 \%$ paraformaldehyde for $10 \mathrm{~min}$ at room temperature, these prepared cells were stained with Hoechst- 33342 for $5 \mathrm{~min}$ at $37^{\circ} \mathrm{C}$ in the dark, and then washed with PBS three times. The Hoechst-stained nuclei characterized by nuclear condensation were visualized by fluorescence microscope.

\section{Immunofluorescent staining}

Cells seeded on cover slips were fixed with $4 \%$ paraformaldehyde, permeabilized in $0.5 \%$ Triton-X100 for $30 \mathrm{~min}$, and blocked with $1 \%$ BSA for $1 \mathrm{~h}$. After that, cells were washed and incubated with anti-mouse iNOS
(Abcam, UK), MR (Abcam, UK) antibodies overnight at $4^{\circ} \mathrm{C}$. Then, cells were incubated with a secondary antibody (Jackson, USA) for $2 \mathrm{~h}$ at room temperature and visualized using an IX70 fluorescence microscope (OLYMPUS, Japan).

\section{Reactive oxygen species (ROS) measurement}

ROS was measured using 2',-7'-dichlorodihydrofluo rescein diacetate (DCFHDA, Sigma). Cells were incubated with DCFHDA at $37^{\circ} \mathrm{C}$ for $30 \mathrm{~min}$ and washed in PBS for $5 \mathrm{~min}$. ROS generation in podocytes was visualized using a fluorescence microscope. The fluorescent intensity was measured by flow cytometry.

\section{ELISA}

The TNF- $\alpha$ level in the CM was detected using ELISA kits (Neobioscience, China) according to the manufacturer's instructions.

\section{Western blot}

The total proteins extracted from the renal cortex and cells were separated by sodium dodecyl sulfate -polyacrylamide gel electrophoresis (SDS-PAGE) and transferred to a nitrocellulose membrane. Nonspecific antibody binding was blocked by a preincubation of the membranes in $1 \times$ TBS containing $5 \%$ skim milk for $1 \mathrm{~h}$ at room temperature. The membranes were incubated overnight with primary antibodies against iNOS (Santa, USA), MR (Abcam, UK), cleaved caspase-3 (Cell Signaling Technology, USA), p-p38MAPK, p38MAPK (Cell Signaling Technology, USA) at $4^{\circ} \mathrm{C}$ followed by incubation with horse reddish peroxidase (HRP) conjugated secondary antibodies for $1 \mathrm{~h}$. Finally, the membranes were visualized with an enhanced chemiluminescence advanced system (GE Healthcare, UK) and captured on X-ray film. Immunoreactive bands were quantified with densitometry using the Image $\mathbf{J}$ software (NIH, USA).

\section{Statistical analysis}

All data were expressed as the mean \pm standard deviation (SD) and analyzed with SPSS 16.0. Statistical differences among different groups were determined by one-way analysis of variance (ANOVA). A difference was considered significant if the $\mathrm{P}$ value was less than 0.05 .

\section{ACKNOWLEDGMENTS}

This study was supported by grants from the National Natural Science Foundation of China (No.81370826 and No.81570612), Medical Key Talents Programs of Jiangsu Province (RC2011124), Clinical Medical Science Technology Special Project of Jiangsu 
Province (No.BL2014080), Fundamental Research Funds for the Central Universities of China and Jiangsu Province Ordinary University Graduate Research Innovation Project (KYLX15_0180).

\section{CONFLICTS OF INTEREST}

None.

\section{REFERENCES}

1. Li JJ, Kwak SJ, Jung DS, Kim JJ, Yoo TH, Ryu DR, Han SH, Choi HY, Lee JE, Moon SJ, Kim DK, Han DS, Kang SW. Podocyte biology in diabetic nephropathy. Kidney Int Suppl. 2007; 72: S36-42.

2. Pagtalunan ME, Miller PL, Jumping-Eagle S, Nelson RG, Myers BD, Rennke HG, Coplon NS, Sun L, Meyer TW. Podocyte loss and progressive glomerular injury in type II diabetes. J Clin Invest. 1997; 99: 342-348.

3. Steffes MW, Schmidt D, McCrery R, Basgen JM. Glomerular cell number in normal subjects and in type 1 diabetic patients. Kidney Int. 2001; 59: 2104-2113.

4. Susztak K, Raff AC, Schiffer M, Bottinger EP. Glucoseinduced reactive oxygen species cause apoptosis of podocytes and podocyte depletion at the onset of diabetic nephropathy. Diabetes. 2006; 55: 225-233.

5. Ryu M, Kulkarni OP, Radomska E, Miosge N, Gross O, Anders HJ. Bacterial CpG-DNA accelerates Alport glomerulosclerosis by inducing an M1 macrophage phenotype and tumor necrosis factor-alpha-mediated podocyte loss. Kidney Int. 2011; 79: 189-198.

6. Chow FY, Nikolic-Paterson DJ, Atkins RC, Tesch GH. Macrophages in streptozotocin-induced diabetic nephropathy: potential role in renal fibrosis. Nephrol Dial Transplant. 2004; 19: 2987-2996.

7. Chow F, Ozols E, Nikolic-Paterson DJ, Atkins RC, Tesch GH. Macrophages in mouse type 2 diabetic nephropathy: correlation with diabetic state and progressive renal injury. Kidney Int. 2004; 65: 116-128.

8. You H, Gao T, Cooper TK, Brian Reeves W, Awad AS. Macrophages directly mediate diabetic renal injury. Am J Physiol Renal Physiol. 2013; 305: F1719-1727.

9. Cao Q, Wang C, Zheng D, Wang Y, Lee VW, Wang YM, Zheng G, Tan TK, Yu D, Alexander SI, Harris DC, Wang Y. IL-25 induces M2 macrophages and reduces renal injury in proteinuric kidney disease. J Am Soc Nephrol. 2011; 22: 1229-1239.

10. Ndisang JF, Jadhav A. Hemin therapy improves kidney function in male streptozotocin-induced diabetic rats: role of the heme oxygenase/atrial natriuretic peptide/adiponectin axis. Endocrinology. 2014; 155: 215-229.

11. Zhang XL, Guo YF, Song ZX, Zhou M. Vitamin D prevents podocyte injury via regulation of macrophage M1/M2 phenotype in diabetic nephropathy rats. Endocrinology. 2014; 155: 4939-4950.

12. Liu Y. Advanced oxidation protein products: a causative link between oxidative stress and podocyte depletion. Kidney Int. 2009; 76: 1125-1127.

13. Spurney RF, Coffman TM. Stressed-out podocytes in diabetes? J Am Soc Nephrol. 2008; 19: 2035-2037.

14. Zheng S, Carlson EC, Yang L, Kralik PM, Huang Y, Epstein PN. Podocyte-specific overexpression of the antioxidant metallothionein reduces diabetic nephropathy. J Am Soc Nephrol. 2008; 19: 2077-2085.

15. Finkel T, Holbrook NJ. Oxidants, oxidative stress and the biology of ageing. Nature. 2000; 408: 239-247.

16. Mittal M, Siddiqui MR, Tran K, Reddy SP, Malik AB. Reactive oxygen species in inflammation and tissue injury. Antioxid Redox Signal. 2014; 20: 1126-1167.

17. Forbes JM, Cooper ME. Mechanisms of diabetic complications. Physiol Rev. 2013; 93: 137-188.

18. Voloboueva LA, Giffard RG. Inflammation, mitochondria, and the inhibition of adult neurogenesis. J Neurosci Res. 2011; 89: 1989-1996.

19. Awad AS, You H, Gao T, Cooper TK, Nedospasov SA, Vacher J, Wilkinson PF, Farrell FX, Brian Reeves W. Macrophage-derived tumor necrosis factor-alpha mediates diabetic renal injury. Kidney Int. 2015; 88: 722-733.

20. Pawluczyk IZ, Harris KP. Transforming growth factor-beta suppresses macrophage-induced mesangial cell fibronectin expression. Kidney Int. 2001; 60: 533-542.

21. Lange-Sperandio B, Fulda S, Vandewalle A, Chevalier RL. Macrophages induce apoptosis in proximal tubule cells. Pediatr Nephrol. 2003; 18: 335-341.

22. Yang Z, Xie H, He D, Li L. Infiltrating macrophages increase RCC epithelial mesenchymal transition (EMT) and stem cell-like populations via AKT and mTOR signaling. Oncotarget. 2016; 7: 44478-44491. doi: 10.18632/ oncotarget.9873.

23. Zhang XL, Selbi W, de la Motte C, Hascall V, Phillips AO. Bone morphogenic protein-7 inhibits monocyte-stimulated TGF-beta1 generation in renal proximal tubular epithelial cells. J Am Soc Nephrol. 2005; 16: 79-89.

24. Patrakka J, Tryggvason K. New insights into the role of podocytes in proteinuria. Nat Rev Nephrol. 2009; 5: 463-468.

25. Wang Y, Cao Q, Zheng G, Lee VW, Zheng D, Li X, Tan TK, Harris DC. By homing to the kidney, activated macrophages potently exacerbate renal injury. Am J Pathol. 2008; 172: 1491-1499.

26. Lindblom R, Higgins G, Coughlan M, de Haan JB. Targeting mitochondria and reactive oxygen species-driven pathogenesis in diabetic nephropathy. Rev Diabet Stud. 2015; 12: 134-156.

27. Schiffer M, Bitzer M, Roberts IS, Kopp JB, ten Dijke P, Mundel P, Bottinger EP. Apoptosis in podocytes induced by TGF-beta and Smad7. J Clin Invest. 2001; 108: 807-816. 
28. Kalantarinia K, Awad AS, Siragy HM. Urinary and renal interstitial concentrations of TNF-alpha increase prior to the rise in albuminuria in diabetic rats. Kidney Int. 2003; 64: 1208-1213.

29. Navarro JF, Mora C, Muros M, Garcia J. Urinary tumour necrosis factor-alpha excretion independently correlates with clinical markers of glomerular and tubulointerstitial injury in type 2 diabetic patients. Nephrol Dial Transplant. 2006; $21: 3428-3434$.

30. Omote K, Gohda T, Murakoshi M, Sasaki Y, Kazuno S, Fujimura T, Ishizaka M, Sonoda Y, Tomino Y. Role of the TNF pathway in the progression of diabetic nephropathy in KK-A(y) mice. Am J Physiol Renal Physiol. 2014; 306: F1335-1347.

31. Baud L, Oudinet JP, Bens M, Noe L, Peraldi MN, Rondeau E, Etienne J, Ardaillou R. Production of tumor necrosis factor by rat mesangial cells in response to bacterial lipopolysaccharide. Kidney Int. 1989; 35: 1111-1118.

32. Lai KN, Leung JC, Chan LY, Saleem MA, Mathieson PW, Lai FM, Tang SC. Activation of podocytes by mesangialderived TNF-alpha: glomerulo-podocytic communication in IgA nephropathy. Am J Physiol Renal Physiol. 2008; 294: F945-955.

33. Ikezumi Y, Suzuki T, Karasawa T, Kawachi H, NikolicPaterson DJ, Uchiyama M. Activated macrophages downregulate podocyte nephrin and podocin expression via stress-activated protein kinases. Biochem Biophys Res Commun. 2008; 376: 706-711. 